\title{
Study on Microstructure Effect of Carbon Black Particles in Filled Rubber Composites
}

\author{
Li Hong Huang $\mathbb{D}^{1,2}$ Xiaoxiang Yang $\mathbb{D}^{1,3}$ and Jianhong Gao ${ }^{1,3}$ \\ ${ }^{1}$ College of Chemical Engineering, Fuzhou University, Fuzhou, Fujian 350108, China \\ ${ }^{2}$ Fuzhou University Zhicheng College, Fuzhou, Fujian 350002, China \\ ${ }^{3}$ Quanzhou Normal University, Quanzhou, Fujian 362000, China
}

Correspondence should be addressed to Xiaoxiang Yang; yangxx@fzu.edu.cn

Received 9 June 2018; Revised 2 August 2018; Accepted 8 August 2018; Published 11 October 2018

Academic Editor: György Bánhegyi

Copyright ( 2018 Li Hong Huang et al. This is an open access article distributed under the Creative Commons Attribution License, which permits unrestricted use, distribution, and reproduction in any medium, provided the original work is properly cited.

The cross sections of blended natural/styrene-butadiene (NSBR) composites filled with different volume fractions of carbon particles were observed using a Quanta 250 scanning electron microscope. In addition, the sizes and distributions of the carbon particles were analyzed using Nano Measurer. A two-dimensional representative volume element model (RVE) for a rubber composite reinforced with circular carbon particles was established, and the uniaxial tensile behaviors of polymer nanocomposites with different particle size distribution patterns were simulated using the ABAQUS software. The results showed the following. (1) For the random models, if the difference of particle size was larger and particle distance was closer, stress distribution would be denser as well as the stress concentration would become greater. However, if the difference of particle size was small, for the case of same particle volume fraction, the particle size has little influence on the macromechanical properties whether the average size is large or small. (2) The correlation between the volume fraction and distribution of the carbon particles revealed that when the volume fraction of carbon black particles was larger than $12 \%$, clusters between carbon particles in the polymer nanocomposites could not be avoided and the modulus of the composites increased with an increase in the cluster number.

\section{Introduction}

As a nanoparticle enhancer, carbon black has been widely used to improve the strength and toughness of polymer nanocomposites $[1,2]$. The reinforcing effect mainly depends on the size, shape, and dispersion degree of the particles in the matrix, as well as the interface adhesion between the particle and matrix. The mixing process for carbon black-filled rubber includes four stages: mixing, dispersing, blending, and plasticizing. After the mixing process, the carbon black particles are fully mixed and then dispersed to form new polycarbon particles with a size of $0.2 \mu \mathrm{m}$. The size of the carbon black particles is changed by the mixing process. The bonding and adsorption degree between the carbon black particles and matrix depend on the new size of the carbon particles [3]. Additionally, because of the limited technological conditions and interaction of the particles' Van der Waals forces, carbon black particles will form a large number of aggregates with the nonuniform structure filler. The shear modulus and elastic modulus of the rubber will be greatly changed according to the differences in the concentration and number of carbon black particles. When the distribution of the carbon black in the composition is bad, the aggregates will increase and the rubber modulus will decrease, which is the Payne effect [4].

Finite element modeling can be used to explain the mechanical properties of the polymer composites. These models are used to reveal the concrete features of the nanostructure, enhancer-matrix interactions, and debonding on the macro- and microstructure. Because of the complexity of the calculations, finite element modeling provides a geometry-based periodic unit cell model called a representative volume element (RVE) to analyze relatively small volumes and deformations, instead of large deformations and complex boundary conditions. The single particle model $[5-7]$ and multiparticle model $[8,9]$ have been widely used to 
TABLE 1: Ingredients of carbon black-filled NSBR composites.

\begin{tabular}{lcccccccc}
\hline Rubber & NR & SBR & N220 & ZnO & Octadecanoic acid & Age resister 4020 & Brim stone & Accelerant \\
\hline NSBR1 & 80 & 20 & 0 & 5 & 2 & 0.7 & 0.7 & 2.6 \\
NSBR3 & 80 & 20 & 20 & 5 & 2 & 2 & 0.7 & 2.6 \\
NSBR4 & 80 & 20 & 30 & 5 & 2 & 0.7 & 2.6 & 1.3 \\
NSBR6 & 80 & 20 & 50 & 5 & 2.6 & 1.3 \\
\hline
\end{tabular}
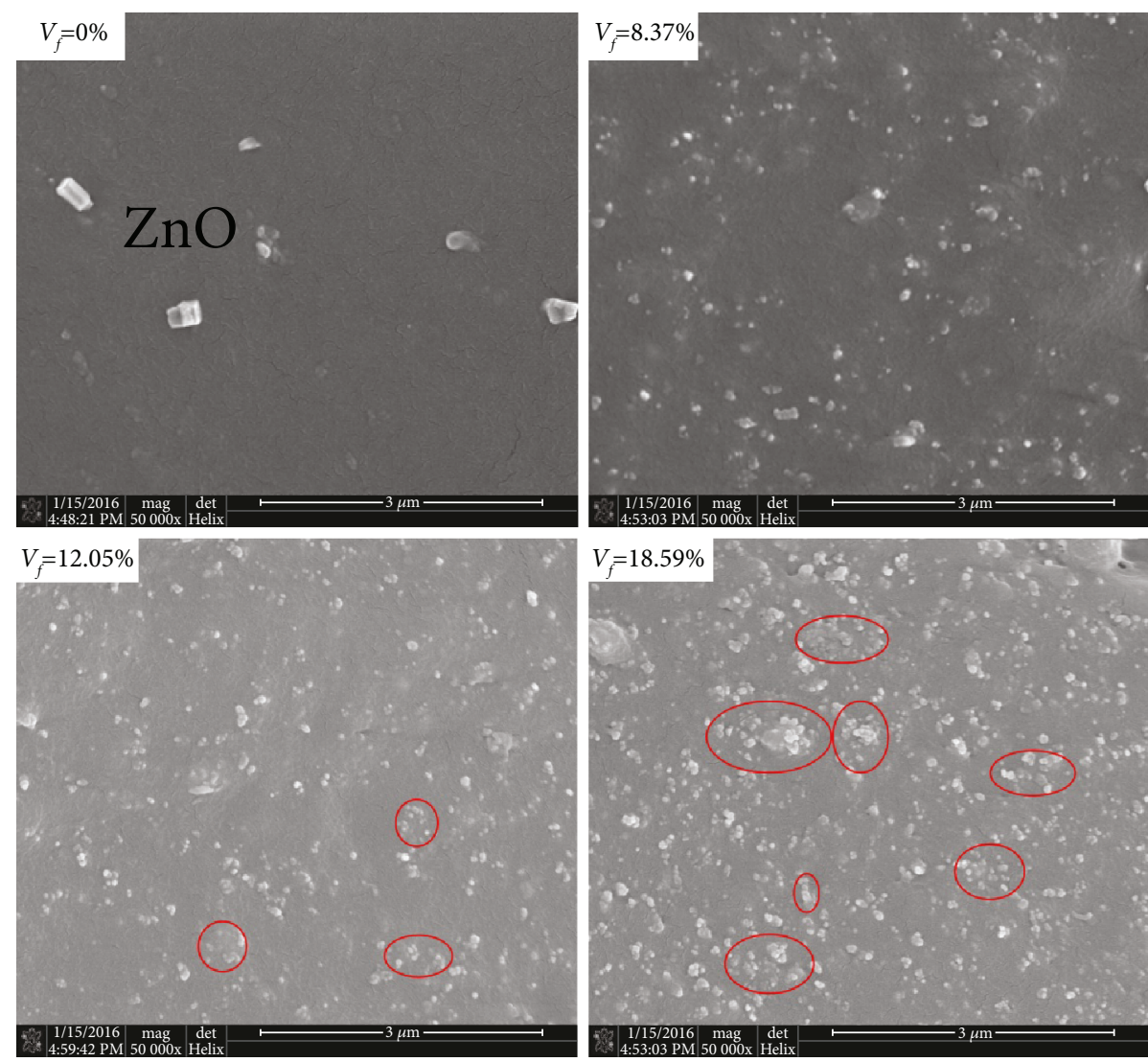

FIGURE 1: SEM photos of rubber composites with different carbon black volume fractions.

analyze the mechanical behaviors of polymer composites. The particle random model [10] and random sequential adsorption model [11] are often used for particle-filled composites. They can effectively embody the macroscopic mechanical properties but ignore the effect of the real mesostructure particle distribution. The new size of the carbon black particles and the number and size of the aggregates in polymer nanocomposites have a great impact on the mechanical properties of the composites.

In the present work, scanning electron microscope (SEM) observations of natural/styrene-butadiene blend rubber (NSBR) composites filled with different volume fractions of carbon particles were performed, as reported in the next section. The distribution of the carbon black particles and whether they are clustered in the polymer nanocomposites were investigated using Nano Measurer, as discussed in Section 3. Structural Modeling presents the results of a numerical simulation using the ABAQUS software, where the results for different carbon structure RVE models are discussed and compared. At the same time, the mechanical properties of the blends were studied. The final section presents the conclusions.

\section{SEM Analysis}

2.1. Rubber Ingredient. In order to investigate the mixing of rubber composites with different carbon black volume fractions, four kinds of specimens were prepared according to the GB528-2009 standard. The ingredients listed in Table 1 show the use of $0,20,30$, and $50 \mathrm{phr}$ of carbon black in the composites, corresponding to filler volume fractions of $0 \%, 8.37 \%, 12.05 \%$, and $18.59 \%$, respectively.

2.2. SEM Analysis. A morphological study was carried out using the Quanta 250 SEM. The test specimens came from the same batch of rubber. First, they were cooled down to the glass transition temperature by liquid nitrogen. They were then cut into two parts with smooth surfaces in the frozen state, and the fracture surface morphological structures were observed after the application of special spray 


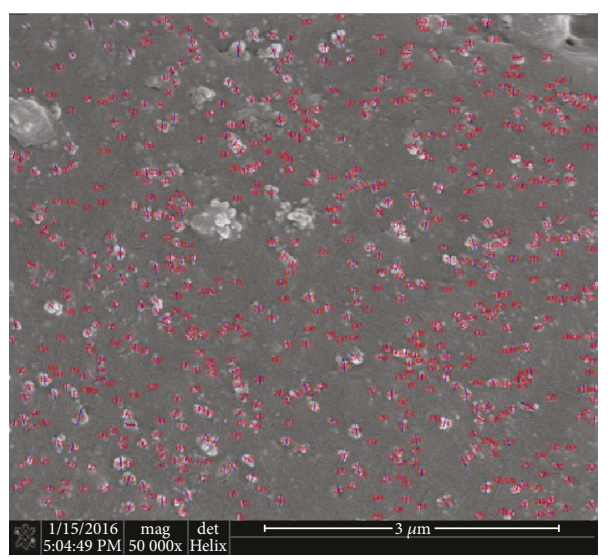

(a) Particles except clusters

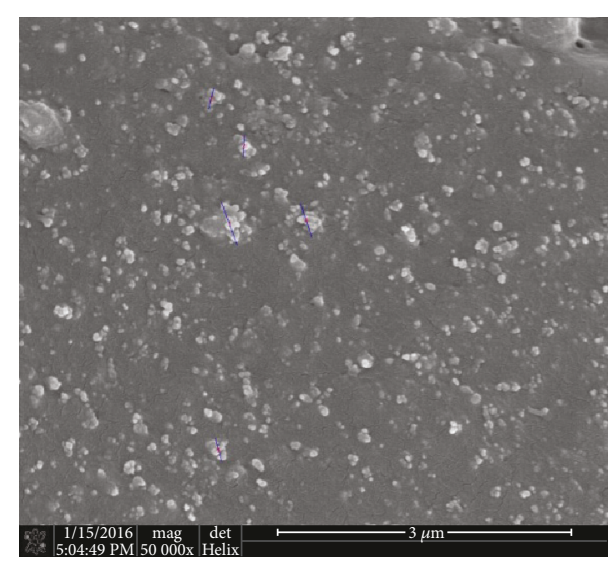

(b) Clusters

FIgURE 2: Particle size and number analysis of NSBR6 rubber.

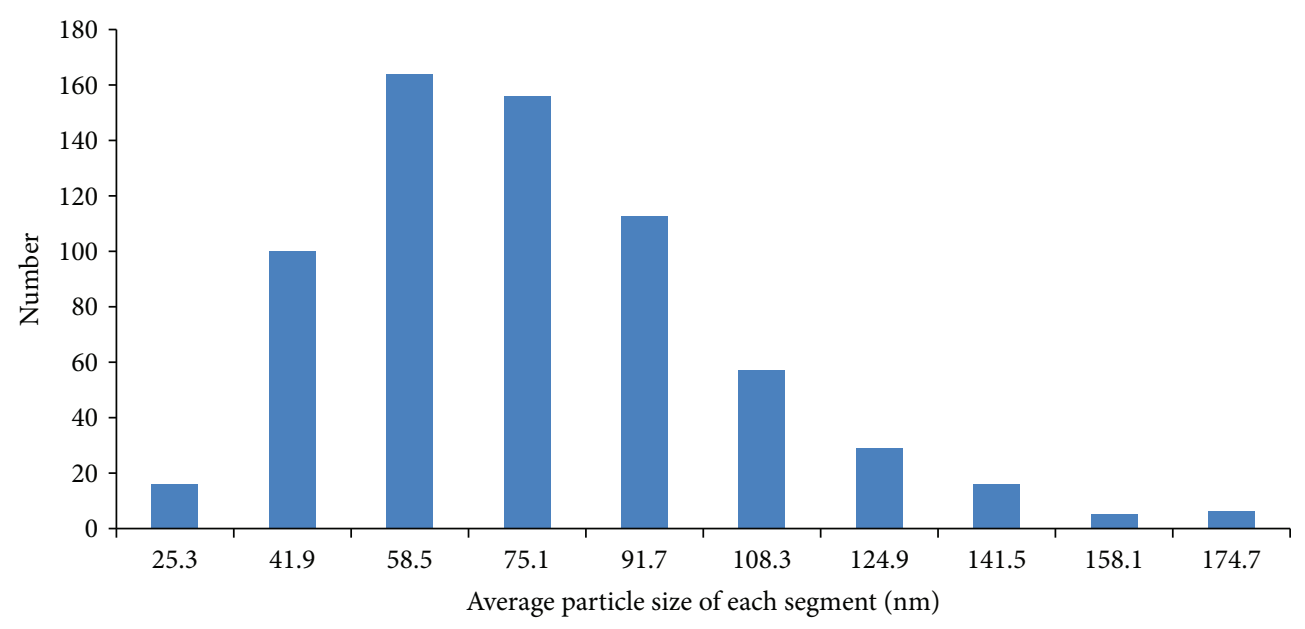

FIgURE 3: Particle size and diameter statistics of NSBR6 rubber.

gold craft paint. In addition, the shape and morphological distribution of the carbon black and whether defects such as holes or cracks existed were observed. The results of the SEM observations of the four kinds of rubber composites with different carbon black volume fractions are shown in Figure 1.

As seen in the SEM image in Figure 1, the two kinds of rubber matrixes, the natural rubber and styrenebutadiene rubber with no carbon black particles filled, are well mixed. The white particles in Figure $1\left(V_{\mathrm{f}}=0 \%\right)$ are $\mathrm{ZnO}$, which had an average size of $200 \mathrm{~nm}$. When the filler volume fraction is approximately $8.37 \%$, the added carbon black (N220) is uniformly dispersed and the particle size is consistent, except for the $\mathrm{ZnO}$. When the filler volume fraction is approximately $12.05 \%$, the carbon black particles are uniformly dispersed, while a small amount of carbon black particles is aggregated. When the filler volume fraction is approximately $18.59 \%$, aggregation appears, with most of the aggregates are small clusters or multiparticle agglomerations. This is a result of the increase in the carbon black volume fraction. In order to discuss the effects of the carbon black morphological distribution and shape on the macroscopic mechanical properties of rubber composites, the particle size distribution will be investigated statistically.

\section{Particle Size and Distribution Statistics}

Figure 2 shows the particle size and distribution of the carbon black particles of NSBR6 found using Nano Measurer. The statistical results for all the particles except clusters are given in Figure 2(a), with those for the clusters are provided in Figure 2(b). As shown in Figure 3 and Table 2, the average particle size of all the carbon black particles in the SEM picture is $75.88 \mathrm{~nm}$ and it is composed of 662 different particles. To investigate the influence of different diameters on the structure, different diameters were counted in ten segments with various sizes and numbers in the range of 17.45$182.15 \mathrm{~nm}$. The average particle sizes of the clusters are $250-460 \mathrm{~nm}$.

\section{Structural Modeling}

4.1. RVE Size Determination and Boundary Conditions. The particle-filled composite was approximately regarded as a periodic set of RVEs, and the mesomechanical properties of 
TABle 2: Statistics results for particle size.

\begin{tabular}{lcccccccccc}
\hline $\begin{array}{l}\text { Diameter } \\
\text { range }(\mathrm{nm})\end{array}$ & $17-33.6$ & $33.6-50.2$ & $50.2-66.8$ & $66.8-83.4$ & $83.4-100$ & $100-116.6$ & $116.6-133.2$ & $133.2-149.8$ & $149.8-166.4$ & $166.4-183$ \\
$\begin{array}{l}\text { Average particle } \\
\text { size (SEM) (nm) }\end{array}$ & 25.3 & 41.9 & 58.5 & 75.1 & 91.7 & 108.3 & 124.9 & 141.5 & 158.1 & 174.7 \\
$\begin{array}{l}\text { Particle number } \\
\text { (SEM) }\end{array}$ & 16 & 100 & 164 & 156 & 113 & 57 & 29 & 16 & 5 & 6 \\
$\begin{array}{l}\text { Percentage } \\
\text { Total number }\end{array}$ & $2.4 \%$ & $15.1 \%$ & $24.8 \%$ & $23.6 \%$ & $17.1 \%$ & $8.6 \%$ & $4.4 \%$ & $2.4 \%$ & $0.8 \%$ & $0.9 \%$ \\
$\begin{array}{l}\text { Total average } \\
\text { particle size (nm) }\end{array}$ & & & & & & & 662 & & & \\
\end{tabular}
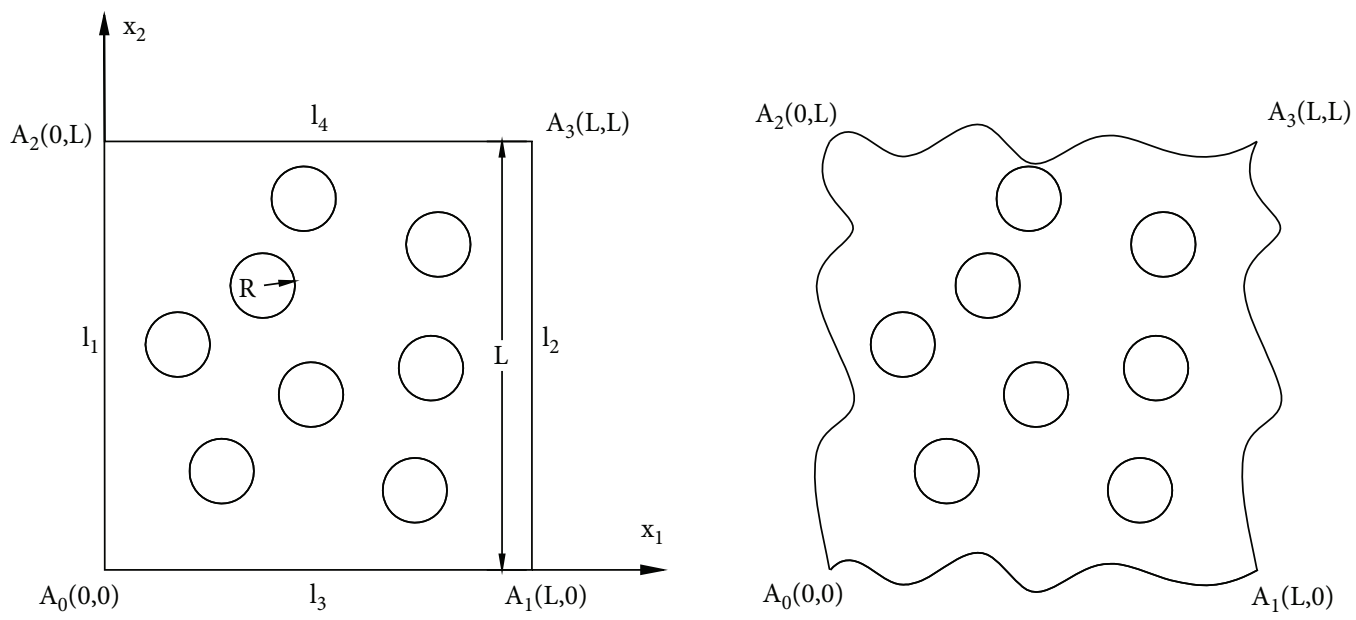

FIgURe 4: Schematic of the 2D RVE model.

the composite were studied by applying periodic boundary conditions to the RVE models. A large number of studies have been effectively performed on the macroscopic properties of composite materials [12-14]. A 3D model is the best choice for numerical simulations. However, because of computational limitations, the 3D multiparticle RVE model could only converge in a very small strain. Therefore, a $2 \mathrm{D}$ RVE model was adopted. Because the area fraction used in the model represented the volume fraction of the composite, the results calculated with the 2D RVE model in ABAQUS deviated from the experimental results. However, the simulation results could be used for a qualitative discussion when the particle size distribution was the same. The size of the RVE elements and the choice of boundary conditions have previously been discussed $[9,15]$.

During the simulation process, the physical properties of the material, elastic modulus, and Poisson's ratio were related to the ratio $L / R$, in which $L$ was the side length of the RVE and $R$ was the radius of a particle. In other words, the particle and RVE sizes determined the reliability of the simulation. When $L / R$ was very small, the numerical solution greatly deviated from the test value. In contrast, when $L / R$ was large enough, the solution was more stable. The two-dimensional RVE model is shown in Figure 4. In order to verify the stability of the model, RVE models with different $L / R$ values were established, with a volume fraction $V_{\mathrm{f}}$ of carbon black particles in NSBR of $10 \%$. The equivalent elastic modulus values

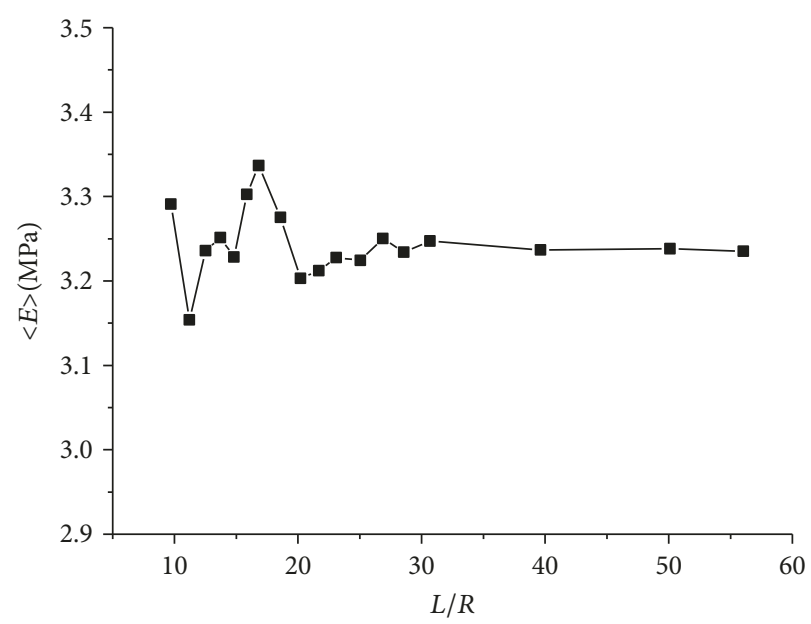

FIgURE 5: Equivalent elastic modulus $\langle E\rangle$ values of NSBR rubber composites $\left(V_{\mathrm{f}}=10 \%\right)$ with different $L / R$ ratios.

of the composite materials $<E>[5]$ in the RVE with different $L / R$ values were calculated.

Figure 5 shows the equivalent elastic modulus $\langle E\rangle$ values of the NSBR rubber composites with different $L / R$ ratios at $V_{\mathrm{f}}=10 \%$. It was found that when the value of $L / R$ was small, the equivalent elastic modulus had a range of 3.15-3.35. When $L / R$ reached to 25 , the equivalent elastic modulus tended to be stable. According to the statistical results, RVE 


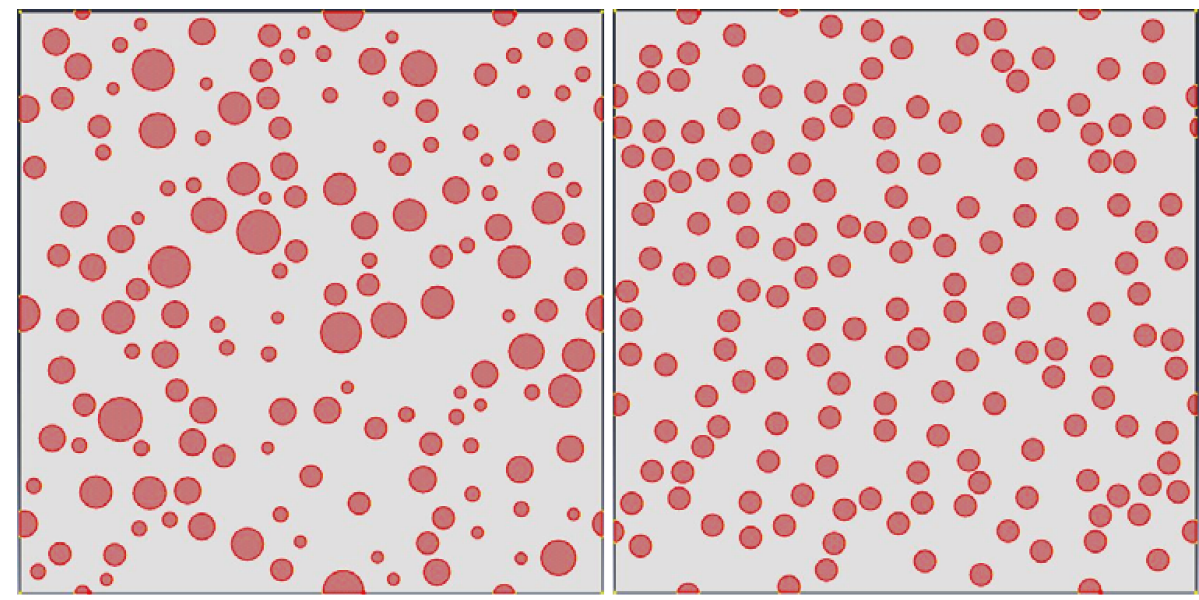

FIgURE 6: Two kinds of RVE random adsorption sequence models.

TABLE 3: Results for particle size and diameter in RVE $\left(V_{\mathrm{f}}=18.59 \%\right)$.

\begin{tabular}{|c|c|c|c|c|c|c|c|c|}
\hline Diameter range $(\mathrm{mm})$ & $33.6-50.2$ & $50.2-66.8$ & $66.8-83.4$ & $83.4-100$ & $100-116.6$ & $116.6-133.2$ & $133.2-149.8$ & $149.8-166.4$ \\
\hline Average particle size (SEM) $(\mathrm{nm})$ & 41.9 & 58.5 & 75.1 & 91.7 & 108.3 & 124.9 & 141.5 & 158.1 \\
\hline Particle number (SEM) & 100 & 164 & 156 & 113 & 57 & 29 & 16 & 5 \\
\hline Average particle size (RVE) (nm) & 40 & 50 & 75 & 90 & 110 & 120 & 140 & 150 \\
\hline Particle number (RVE) & 23 & 40 & 36 & 28 & 13 & 7 & 4 & 2 \\
\hline
\end{tabular}

side length $L$ was on the micrometer scale and $L / R$ had a value greater than 25 .

The periodic boundary conditions must be applied to the RVE model [16]. The nodes on the borders repeat those on the opposite side borders, and the displacements and deformations of the corresponding nodes on two opposite boundaries remain the same. For this purpose, the boundary displacement constraint is considered as follows:

$$
\begin{gathered}
\boldsymbol{u}\left(x_{1}, L\right)=\boldsymbol{u}\left(x_{1}, 0\right)+\left(\boldsymbol{u}_{A_{2}}-\boldsymbol{u}_{A_{0}}\right), \\
\boldsymbol{u}\left(L, x_{2}\right)=\boldsymbol{u}\left(0, x_{2}\right)+\left(\boldsymbol{u}_{A_{1}}-\boldsymbol{u}_{A_{0}}\right),
\end{gathered}
$$

where $\boldsymbol{u}_{A_{i}}$ are the displacement vectors of $A_{i}, i=0,1,2$. Respectively, $l_{1}, l_{2}, l_{3}$, and $l_{4}$ are the borders of $x_{1}=0, x_{1}=L$, $x_{2}=0$, and $x_{2}=L$, as shown in Figure 4 . The displacement vector of the points in $l_{i}$ are $\boldsymbol{u}_{l_{i}}$, which satisfy the following conditions:

$$
\begin{aligned}
& \boldsymbol{u}_{l_{4}}=\boldsymbol{u}_{l_{3}}+\left(\boldsymbol{u}_{A_{2}}-\boldsymbol{u}_{A_{0}}\right), \\
& \boldsymbol{u}_{l_{2}}=\boldsymbol{u}_{l_{1}}+\left(\boldsymbol{u}_{A_{1}}-\boldsymbol{u}_{A_{0}}\right) .
\end{aligned}
$$

\subsection{Structural Modeling}

4.2.1. Modeling. The geometric model of the $2 \mathrm{D}$ RVE of the composite material was established according to the statistical results for the carbon black in the NSBR6 rubber presented in Figure 3 and Table 2. The side length of the RVE $L=2 \mu \mathrm{m}$, because the sizes of RVE unit and SEM unit are different; in order to ensure the number of particles is integer, the average diameter of particles is slightly different from that
TABLE 4: Comparison of two kinds of models.

\begin{tabular}{lcc}
\hline & $\begin{array}{c}\text { Number of } \\
\text { particles }\end{array}$ & $\begin{array}{c}\text { Number of } \\
\text { grids }\end{array}$ \\
\hline $\begin{array}{l}\text { Same particle size model } \\
\begin{array}{l}\text { Different particle size } \\
\text { model }\end{array}\end{array}$ & 168 & 52683 \\
\hline
\end{tabular}

of SEM but make sure that the area fraction is the same and the numerical results are not affected. For the same volume fraction $V_{\mathrm{f}}=18.59 \%$, the numbers of particles with different diameters in the RVE are calculated and listed in Table 2. To improve the convergence and accuracy, the maximum and minimum particle diameters are deleted. The average particle diameter of each segment is set for the different diameters in the RVE, and the ratio $L / R \geq 26.7$ for RVE side length $L=2$ $\mu \mathrm{m}$, maximum particle size $D=150 \mathrm{~nm}$, and $R=75 \mathrm{~nm}$. The solution is stable. Two kinds of RVE random adsorption sequence models are shown in Figure 6. For the random adsorption sequence model of the carbon particle diameter segment, the average particle diameter of each segment is listed in Table 3. The average diameter of all particles is $75.88 \mathrm{~nm}$, while the number of particles is 168 when the side length of the RVE is $L=2 \mu \mathrm{m}$ and the volume fraction is $18.59 \%$. The number of particles and mesh generation of the two kinds of models is shown in Table 4.

The main mechanical parameters used in the model are as follows. Carbon black was supposed to be a linear and elastic material. Its elastic modulus $E_{\mathrm{f}}=500 \mathrm{MPa}$, and Poisson's ratio $\mu_{\mathrm{f}}=0.3$ [5]. The parameters of the experimental data are fitted based on the multistep relaxation stretching cyclic 


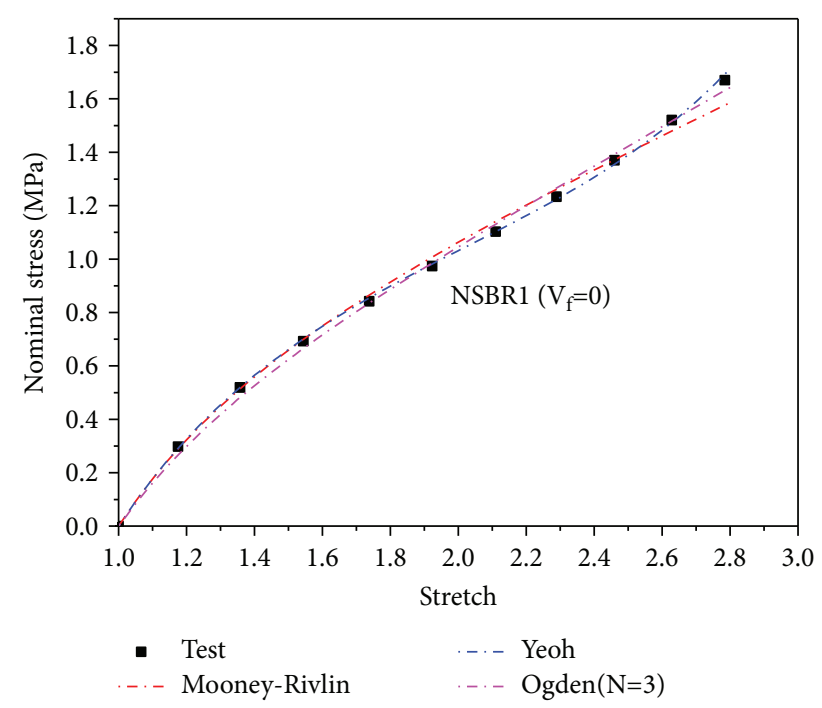

FIGURE 7: Fitting results of some constitutive models for tensile experimental data of NSBR1.

TABLE 5: Material parameters of Yeoh models (NSBR1).

\begin{tabular}{lccc}
\hline Rubber & $C_{10}(\mathrm{MPa})$ & $C_{20}(\mathrm{MPa})$ & $C_{30}(\mathrm{MPa})$ \\
\hline NSBR1 & 0.652 & -0.0119 & 0.001367 \\
\hline
\end{tabular}

loading and unloading test of the NSBR blends at room temperature using the automatic control testing machine of SHIMADZU AG-plus $50 \mathrm{kN}$, with the results shown in Figure 7. The Yeoh constitutive model is adopted for the matrix material, and the material parameters are summarized in Table 5. The Yeoh strain energy function under uniaxial extension is given by (3). The adaptive grid type is the quadratic complete integral of the plane strain eight-node quadrilateral hybrid unit CPE8H for the matrix and CPE8 for the carbon. Simulated uniaxial tension tests are performed using the periodic boundary conditions.

$$
W_{\text {Yeoh }}=C_{10}\left(I_{1}-3\right)+C_{20}\left(I_{1}-3\right)^{2}+C_{30}\left(I_{1}-3\right)^{3} \text {. }
$$

In order to investigate these different random causes, the same analysis of the different random models was performed for a volume fraction of $18.59 \%$. All of the stress-strain curves of the composites (Figures 8 and 9) show that these results are almost coincident and the random error is small.

4.2.2. Results. Figures 10(a) and 10(b) show the distribution of the Mises stress isolines with the different particle size random model and same particle size random model in the case of the uniaxial tensile test with displacement $x=2 \mu \mathrm{m}$; models are created with four RVEs in the $x$ and $y$ directions in an RVE array. As seen in Figure 9, the stress isoline at the joint boundary of two adjacent RVEs is continuous and closed in the loading process. In other words, the stress isoline of the boundary in the RVE is continuous, which satisfies the uniform stress in the boundary of the corresponding node of the RVE and conforms to the stress continuity of the periodical structure.

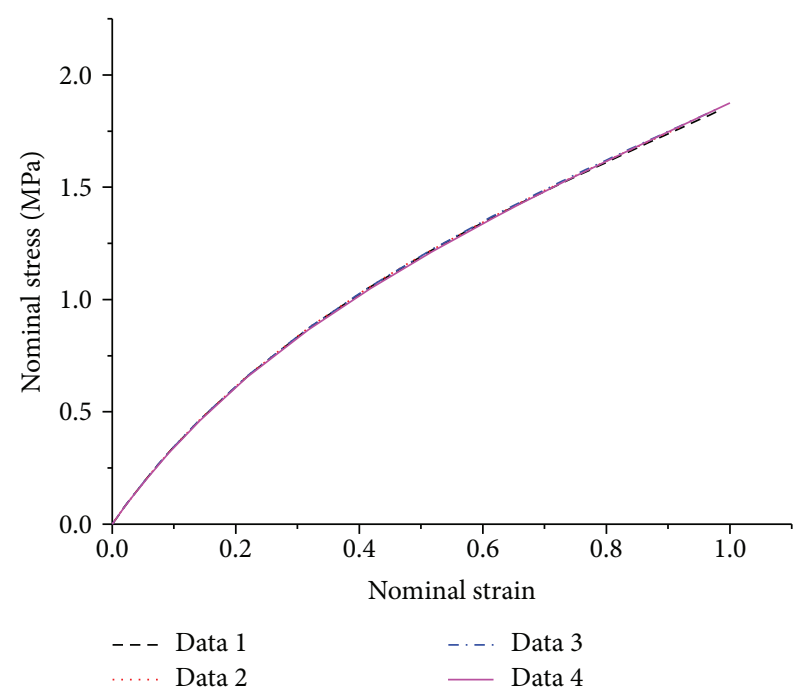

FIgURE 8: Stress-strain curves of different stochastic models with same particle size for NSBR6 in case of uniaxial tensile.

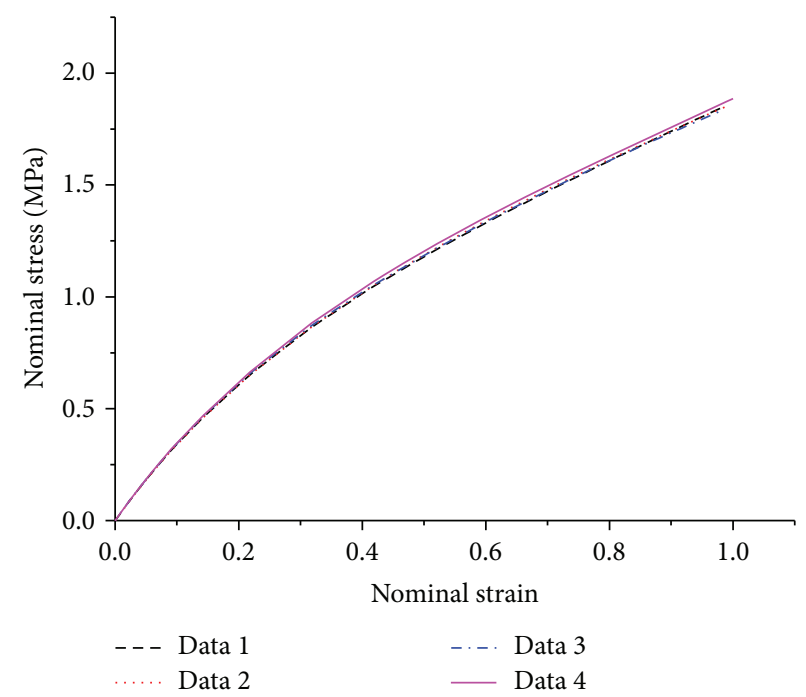

Figure 9: Stress-strain curves of different stochastic models with different particle sizes for NSBR6 in case of uniaxial tensile.

Figures 11(a) and 11(b) show the deformations and Mises stress isolines with the different particle size random model and same particle size random model in uniaxial tensile with displacement $x=2 \mu \mathrm{m}$. By comparison with Figure 6, it can be found that the Mises stress distributions of the two kinds of models are not uniform. In the dense particle distribution area, the stress concentration is more obvious and the stress isolines are denser when the particle positions are closer. Because of the different particle sizes, the stress concentration is very serious in the dense region of the large particles. Smaller particles show more diversity in the particle size difference and a smaller stress gradient. In the same particle size random model, the stress isolines are sparse, which indicates that the stress concentration of the different particle size stochastic models is more obvious than that of the same particle size random models. 


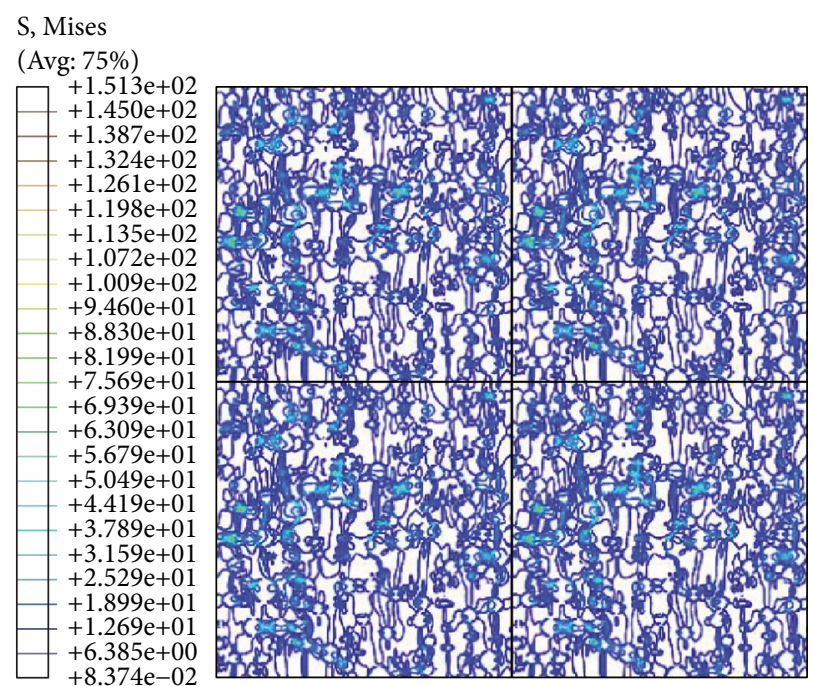

(a)
S, Mises

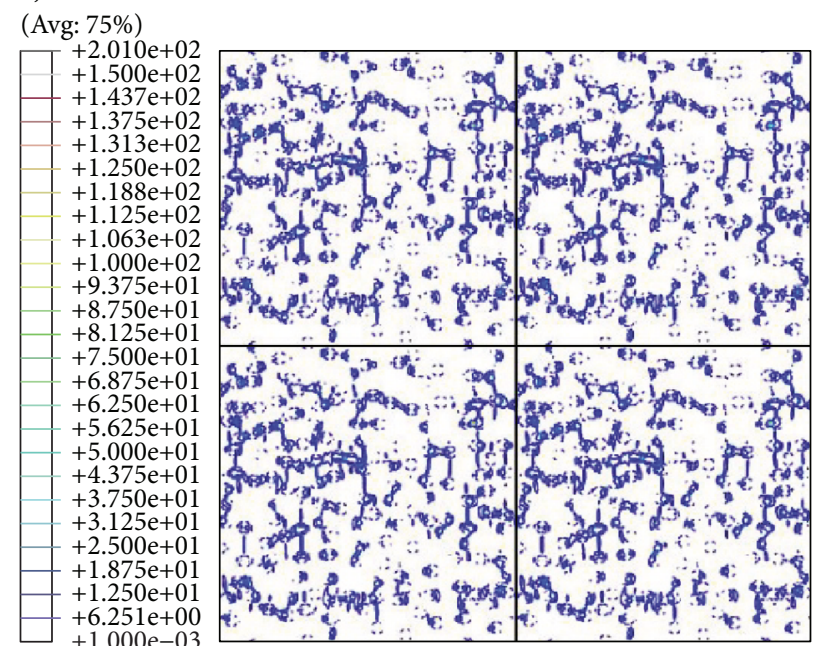

(b)

Figure 10: Distribution of Mises stress in two kinds of RVE models: (a) different particle size random model and (b) same particle size random model.

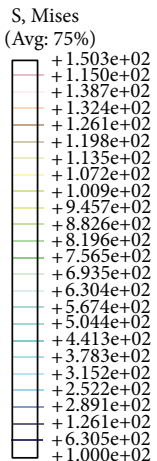

$1.000 \mathrm{e}+02$

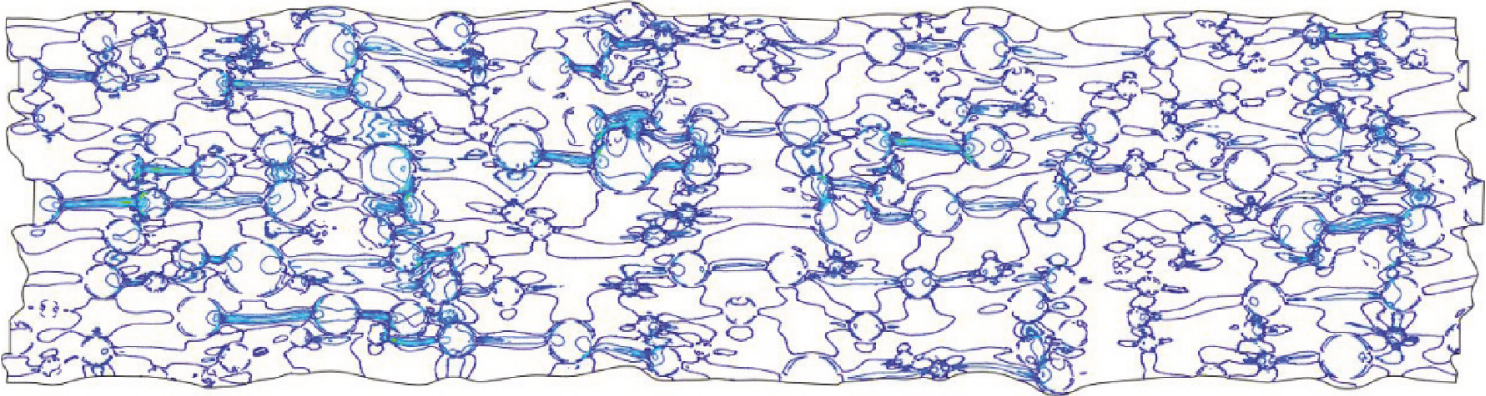

(a)
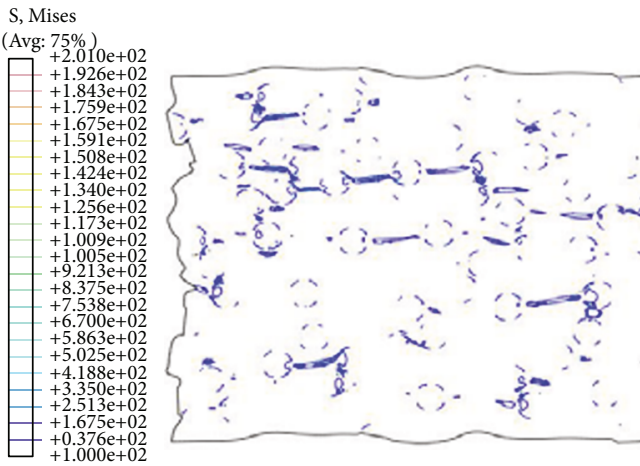

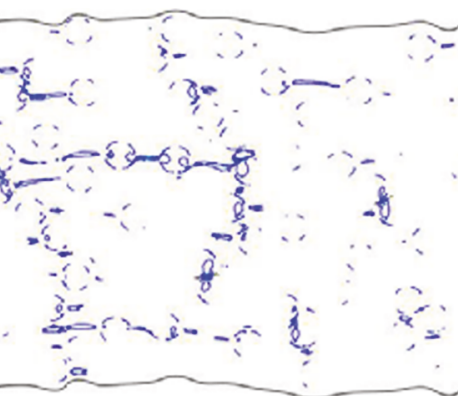

(b)

FIgURE 11: Deformation and distribution of Mises stress in two kinds of RVE models: $(\varepsilon=1)$ (a) different particle size random model and (b) same particle size random model.

Figure 11(a) plots the deformation in the uniaxial tensile state. The stress-deformation relationship between the matrix and particles is complex because of the influence of the distance between the particles and the particle size. It is not difficult to find that a series of shear bands [17] is formed when the $x$ direction is in uniaxial tensile. In the continuous shear zone, the stress isolines in the particles and matrix are dense and the maximum stress lies in it. At the same time, when the dense shear zone appears, the stress concentration will also occur here. When the differences in particle size are greater or a close particle has a larger particle size, the stress shear zone will appear, the stress gradient becomes 


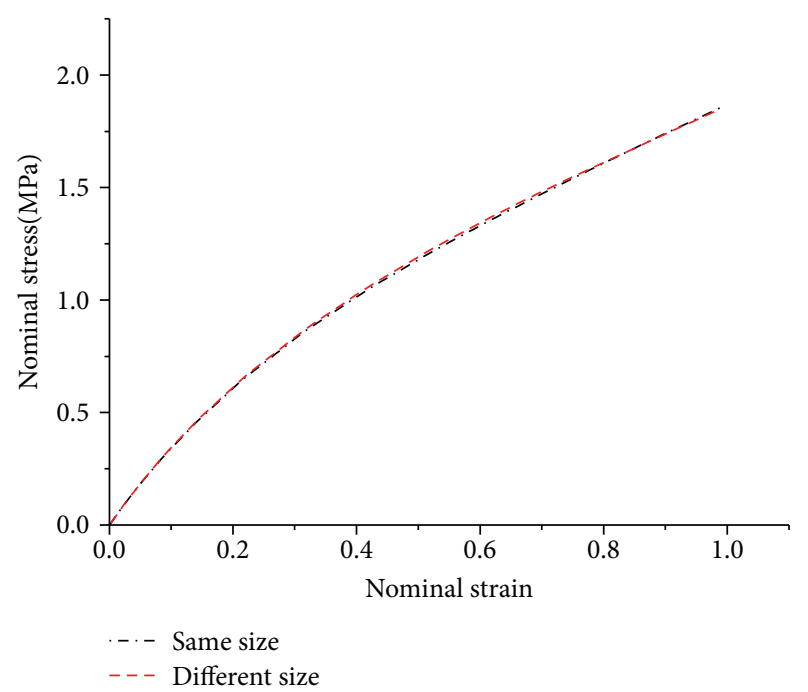

FIGURE 12: Nominal stress-strain curves for two kinds of RVE models.

larger, and the stress concentration is obvious. This indicates that the stress concentration is mainly determined by the differences in the positions and sizes of the particles in the tensile direction and also influenced by the distribution of the particles in the vertical direction.

The deformation is displayed in Figure 11(b). A similar shear band between the particles is formed, but it is not obvious. This is because there is no difference in particle size. In addition, the maximum stress is $151.3 \mathrm{MPa}$ in the different particle size random model, which is located between two similar large particles in the shear band. However, the maximum stress is $201 \mathrm{MPa}$ in the same particle size random model, which is located between the two nearest particles with $x$ direction distance $x=0.0108 \mu \mathrm{m}$. Therefore, it can be concluded that the complex stress-deformation relationship between the matrix and particles mainly depends on the distance between the particles in the stretching direction, the relative position, and the particle size. When the particle distance in the tensile direction is closer, it is easier to form the shear zone in the tensile direction and the larger difference in the particle size will lead to an obvious stress concentration.

The nominal stress-strain curves of the two kinds of models are shown in Figure 12. The macroscopic stressstrain curves of the different random models are basically coincident in the case with the same numbers of meshes and particles. The results show that the particle size difference for the rubber specimen prepared in this study will not have a great influence on the macroscopic tensile properties, but interfacial debonding occurs between the matrix and carbon particles during the tensile process and the stress concentration has a significant effect on the stress-strain after this interfacial debonding.

\subsection{Effects of Different Particle Clusters}

4.3.1. Modeling. Based on the above analysis, the distance between the particles has a great influence on the stress concentration of the composites. Here, the random sequence adsorption (RSA) algorithm [18] is adopted to discuss the distribution pattern of the particles instead of the ordinary random model in the mesomodel because the RSA ensures that the shortest distance between the particles is $\geq 0.07 R$ and the convergence of the calculation is faster. Taking into account the spacing of the particles when they aggregate, the spacing between any two particles in the clusters is set to be the shortest distance $0.07 R$. The average particle size of the clusters is $250-460 \mathrm{~nm}$ (Figure 2), and 19 particles form a cluster. Figure 13 presents the random models of different numbers of clusters. The numbers of clusters and grids of the different cluster models are listed in Table 6. The tensile behaviors of the filled rubber with different numbers of clusters will be discussed.

4.3.2. Result. Figure 14 shows the deformation and von Mises stress isoline (same particle size) of the NSBR6 for a nominal strain $\varepsilon=0.4$. The von Mises stress contour in Figure 14 shows that the stress concentrations in composites with clusters are more dramatic than that with no clustered particles in uniaxial tensile. The maximum von Mises stress values are different for different numbers of clusters. Specifically, the maximum stress is $47.82 \mathrm{MPa}$ in the RVE model with no clustered particles, and the maximum stress values are 52.58 MPa, 53.94 MPa, 55.97 MPa, 54.61 MPa, $58.56 \mathrm{MPa}$, and $101.7 \mathrm{MPa}$ in the RVE models with $1,2,3,4,5$, and 6 clusters, respectively. The maximum stress increases with the number of clusters. The contour density of the other particles is much smaller than that of the clusters. At the same time, the stress distribution of particles near the clusters is also affected. The contour distribution of the other particles is narrower when the number of clusters is greater. The stress in the tensile direction of the thin layer rubber matrix between the particles in the clusters is relatively larger, and it is possible for interfacial debonding or cracking to appear.

Figure 15 shows the nominal stress-strain curves of NSBR6 with different numbers of clusters. The curves show that the elastic modulus of the filled rubber slightly increases when the number of clusters is greater. Therefore, for the same strain, the nominal stress increases with the number of clusters. For example, at nominal strain $\varepsilon=0.4$, the nominal stress is $1.026 \mathrm{MPa}$ in the RVE model with no clustered particles and the nominal stress values are $1.035 \mathrm{MPa}, 1.048 \mathrm{MPa}, 1.093 \mathrm{MPa}, 1.128 \mathrm{MPa}, 1.133 \mathrm{MPa}$, and $1.230 \mathrm{MPa}$ in the RVE models with $1,2,3,4,5$, and 6 clusters, respectively. Comparing Figures 13 and 14, it can be concluded that the macroscopic properties of the composites have a meaningful relationship to whether the particles cluster in the material and to the number of clusters. A significant stress concentration in the composites will cause the material to generate microscopic and macroscopic defects, and the convergence will be reduced. Because the results calculated with the 2D RVE model in ABAQUS were deviated from the experimental results as we discussed before, the simulation results were used for a qualitative discussion. The modulus of the calculated curve increased as the number of clusters increased, and experimental results were more close to those from the 

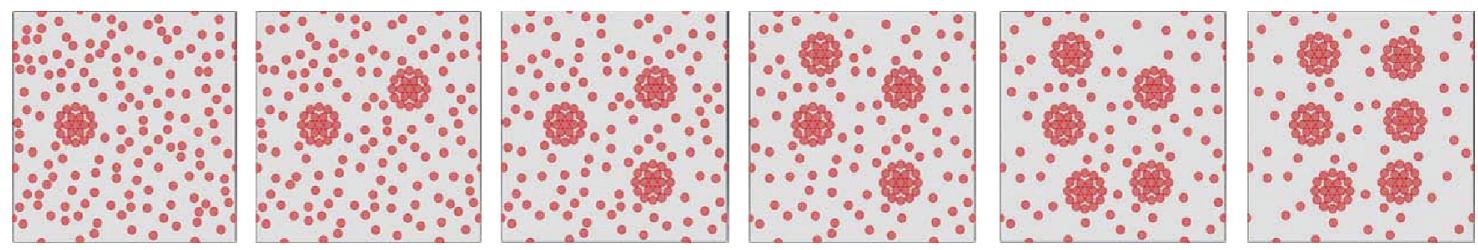

FIGURE 13: Different carbon cluster numbers in same particle size models.

TABLE 6: Grid numbers of different cluster models.

\begin{tabular}{lcccccc}
\hline Size & \multicolumn{5}{c}{ Same particle size } \\
\hline Cluster number & 0 & 1 & 2 & 3 & 4 & 5 \\
Grid number & 52683 & 52818 & 52714 & 52948 & 52930 & 53182 \\
\hline
\end{tabular}

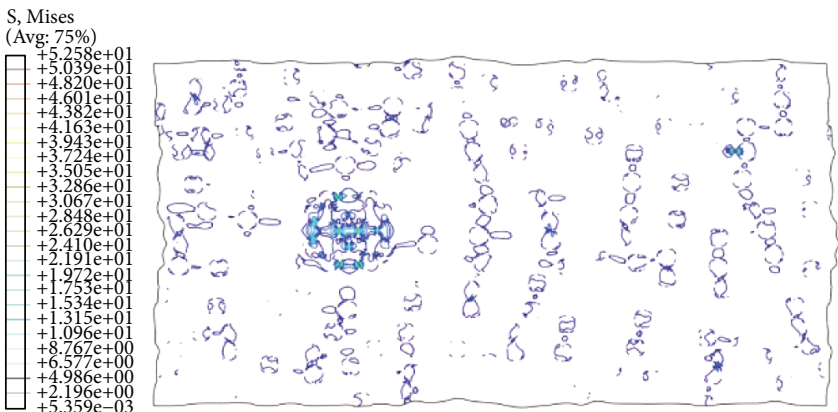

(a)
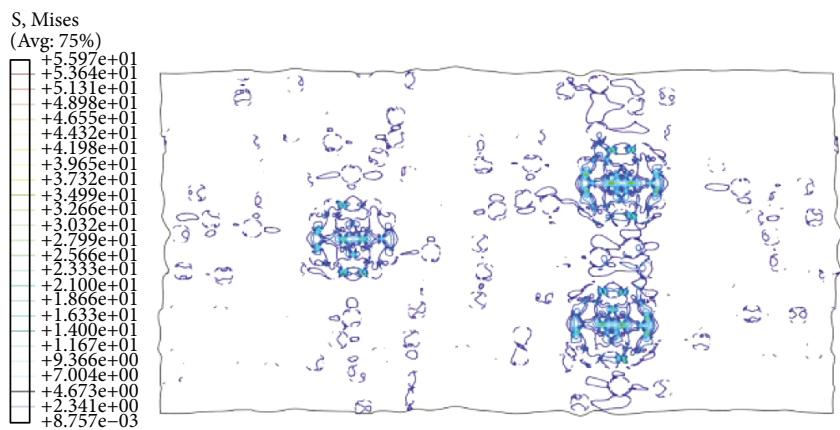

(c)
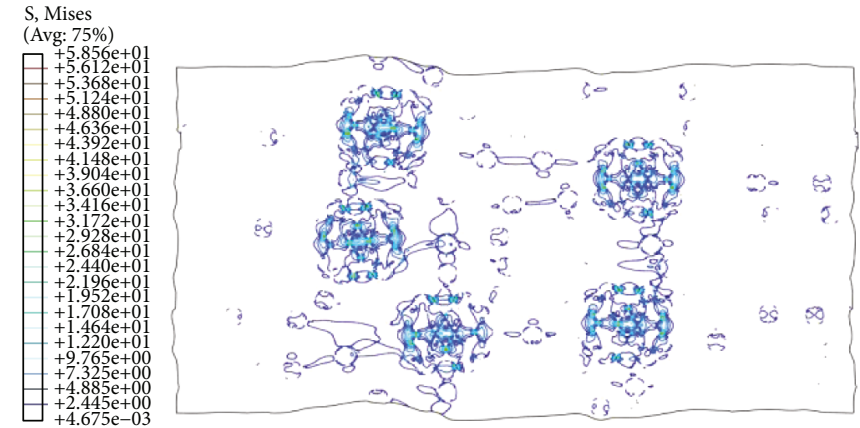

(e)
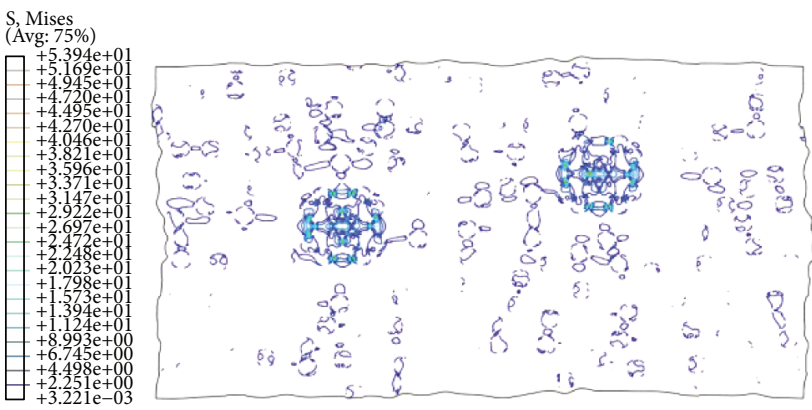

(b)
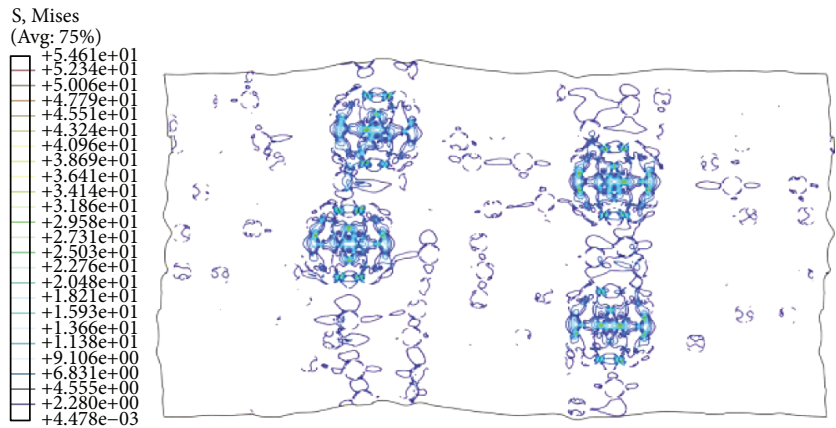

(d)
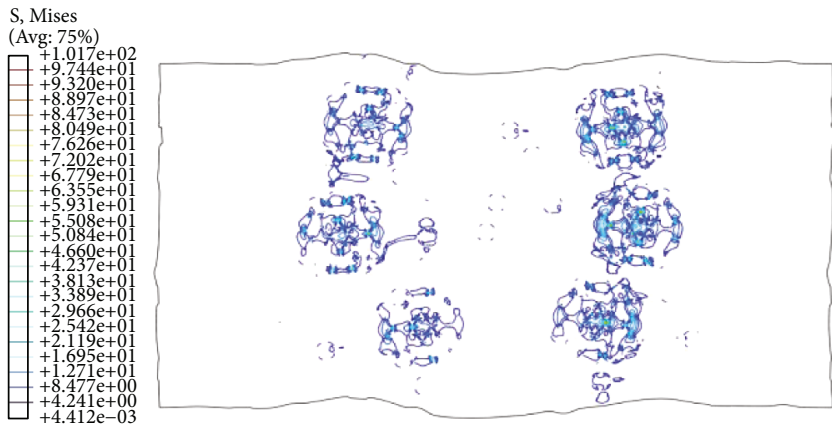

(f)

FIGURE 14: Deformation and distribution of Mises stress in the RVE model with different numbers of clusters ( $\varepsilon=0.4)$ (same size): (a) one cluster, (b) two clusters, (c) three clusters, (d) four clusters, (e) five clusters, and (f) six clusters. 


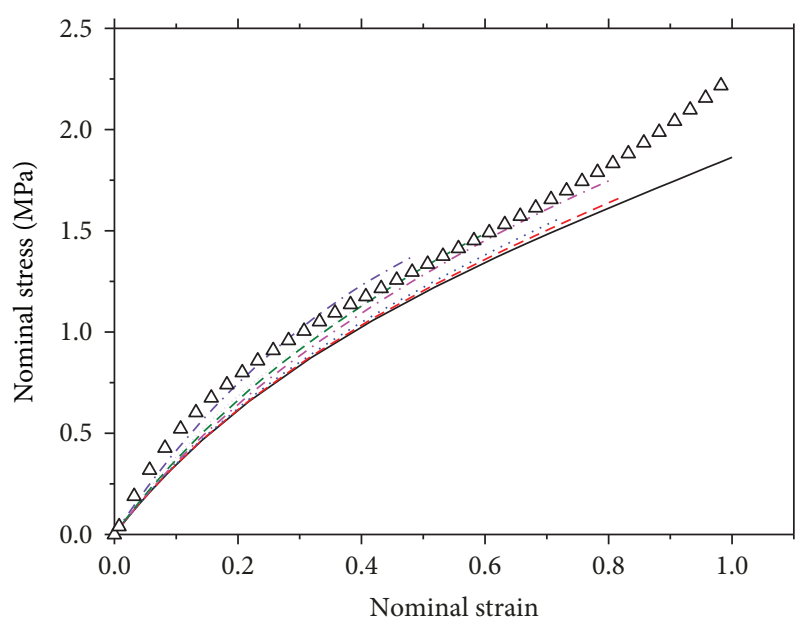

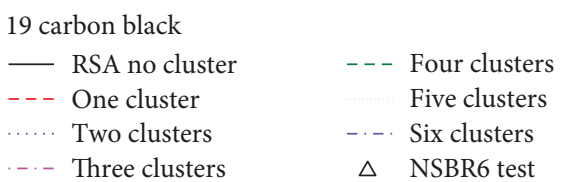

FIGURE 15: Stress-strain curves of NSBR6 with different numbers of clusters (same particle size).

simulations, which took into account clusters with a smaller deviation.

\section{Conclusions}

The SEM results for NSBR composites showed that the carbon black is dispersed more and more unevenly in the mixing process with an increase in the carbon black content. Therefore, the agglomeration phenomenon cannot be neglected when the carbon black N220 particle volume fraction reaches $12 \%$.

For this reason, a combined statistical-numerical approach was adopted to determine the RVE size. When $L$ $I R$ reaches a certain value, the equivalent elastic modulus tends to be stable. In addition, the stress distribution and stress concentration are related to the size, spacing, and relative positions of particles on the mesoscale. However, there is no difference in the macroscopic stress-strain curve.

This is caused by the fact that the material with localized cluster particles loses homogeneity. The simulated results indicated that the elastic modulus of a composite increased slightly when the clusters were taken into account. The macroscopic properties of the composites have a meaningful relationship to the size and amount of the particle clusters. A significant stress concentration would lead to inner defects.

\section{Data Availability}

The data used to support the findings of this study are available from the corresponding author upon request.

\section{Conflicts of Interest}

The authors declare that they have no conflicts of interest.

\section{Acknowledgments}

The support from NSFC (Grant no. 11372074) and Education Science and Technology Project JAT170850 of Fujian Province is appreciated.

\section{References}

[1] B. C. Kim, S. W. Park, and D. G. Lee, "Fracture toughness of the nano-particle reinforced epoxy composite," Composite Structures, vol. 86, no. 1-3, pp. 69-77, 2008.

[2] N. Zheng, Y. Huang, H.-Y. Liu, J. Gao, and Y.-W. Mai, "Improvement of interlaminar fracture toughness in carbon fiber/epoxy composites with carbon nanotubes/polysulfone interleaves," Composites Science and Technology, vol. 140, pp. 8-15, 2017.

[3] B. F. Guan, G. F. Ma, R. M. Wei, and B. Y. Chen, "Rubber fatigue failure model based on the dispersion degree of carbon black in filler rubber," World Rubber Industry, vol. 38, no. 6, pp. 20-23, 2011.

[4] G. Heinrich and T. A. Vilgis, "A statistical mechanical approach to the Payne effect in filled rubbers," Express Polymer Letters, vol. 9, no. 3, pp. 291-299, 2015.

[5] J. S. Bergstrom and M. C. Boyce, "Mechanical behavior of particle filled elastomers," Rubber Chemistry and Technology, vol. 72, no. 4, pp. 633-656, 1999.

[6] Y. Fukahori and W. Seki, "Stress analysis of elastomeric materials at large extensions using the finite element method. Part II. Stress and strain distribution around rigid spherical particles," Journal of Materials Science, vol. 28, no. 16, pp. 4471-4482, 1993.

[7] V. V. Moshev and L. L. Kozhevnikova, "Structural cell of particulate elastomeric composites under extension and compression," International Journal of Solids and Structures, vol. 39, no. 2, pp. 449-465, 2002.

[8] M. D. Rintoul and S. Torquato, "Reconstruction of the structure of dispersions," Journal of Colloid and Interface Science, vol. 186, no. 2, pp. 467-476, 1997.

[9] R. J. M. Smit, W. A. M. Brekelmans, and H. E. H. Meijer, "Prediction of the mechanical behavior of nonlinear heterogeneous systems by multi-level finite element modeling," Computer Methods in Applied Mechanics and Engineering, vol. 155, no. 1-2, pp. 181-192, 1998.

[10] C. Andraud, A. Beghdadi, E. Haslund, R. Hilfer, J. Lafait, and B. Virgin, "Local entropy characterization of correlated random microstructures," Physica A: Statistical Mechanics and its Applications, vol. 235, no. 3-4, pp. 307-318, 1997.

[11] J. LLorca and J. Segurado, "Three-dimensional multiparticle cell simulations of deformation and damage in spherereinforced composites," Materials Science and Engineering: A, vol. 365, no. 1-2, pp. 267-274, 2004.

[12] M. Kulkarni, Finite Element Analysis of 2-D Representative Volume Element, Michigan Technological University, 2012.

[13] Y. Cao, Representative Volume Element (RVE) FiniteElement Analysis (FEA) of Al Metal-Matrix Composites, University of Wisconsin-Milwaukee, 2016.

[14] J. C. Yun and S. J. Park, "Development of representative volume element homogenization model for predicting transversely isotropic elasticity of lithium-ion batteries," Journal of Engineering Materials and Technology, vol. 139, no. 4, article 041008, 2017. 
[15] I. M. Gitman, H. Askes, and L. J. Sluys, "Representative volume: existence and size determination," Engineering Fracture Mechanics, vol. 74, no. 16, pp. 2518-2534, 2007.

[16] R. J. M. Smit, W. A. M. Brekelmans, and H. E. H. Meijer, "Prediction of the large-strain mechanical response of heterogeneous polymer systems: local and global deformation behaviour of a representative volume element of voided polycarbonate," Journal of the Mechanics and Physics of Solids, vol. 47, no. 2, pp. 201-221, 1999.

[17] K. Qiu, Y. P. Jiang, X. P. Shi, L. G. Sun, and Q. Q. Wu, "Microstructure effect of metallic glass matrix composites," Acta Materiae Compositae Sinica, vol. 34, no. 6, pp. 1316-1324, 2017.

[18] Q. Li and X. Yang, “Three dimensional numerical simulation for mechanical behavior of carbon black filled rubber composites," Chinese Journal of Solid Mechanics, vol. 34, no. 6, pp. 541-549, 2013. 


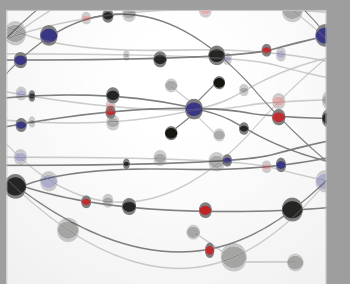

The Scientific World Journal
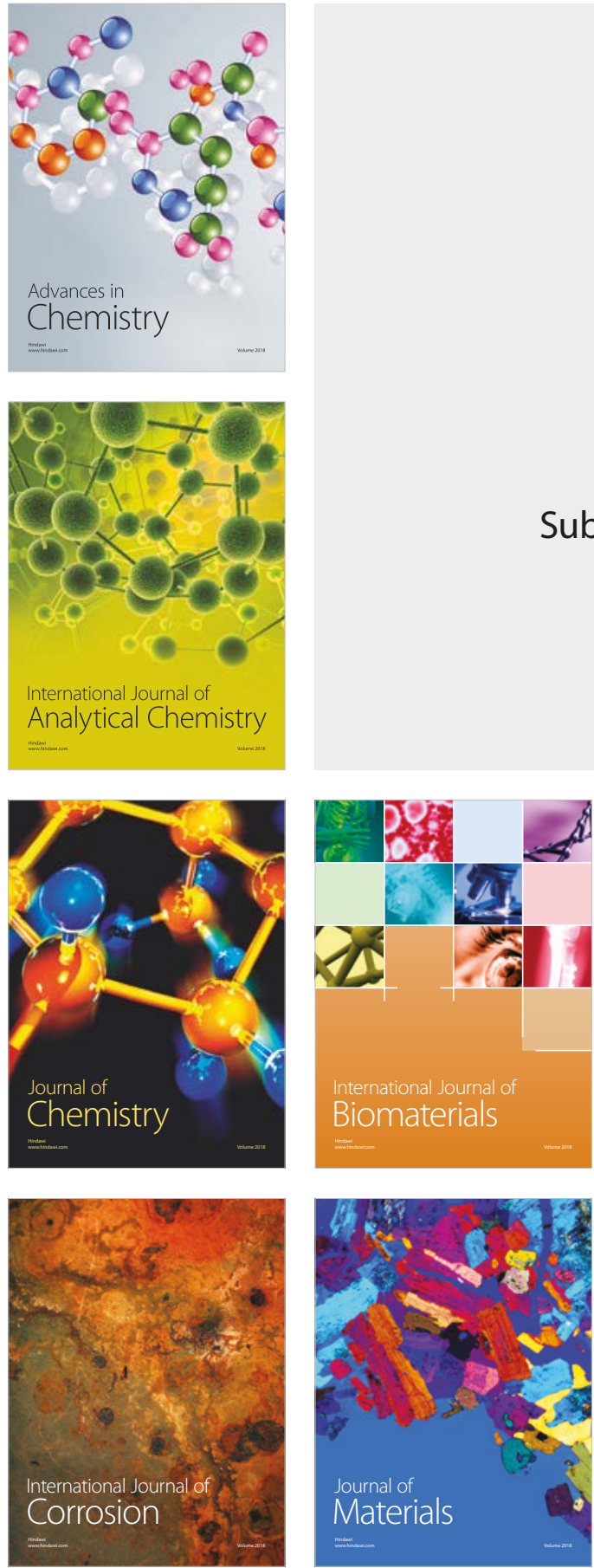

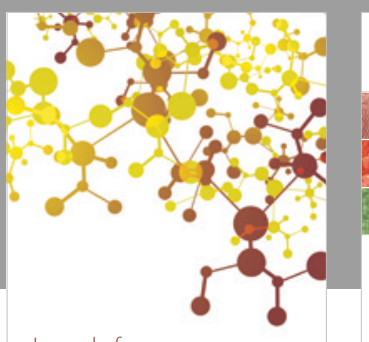

Journal of

Applied Chemistry
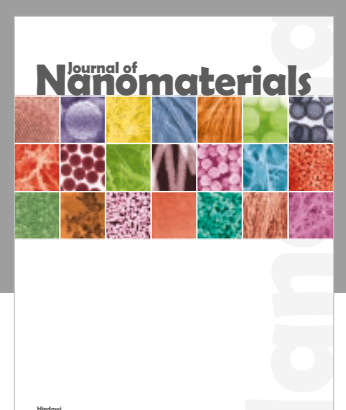

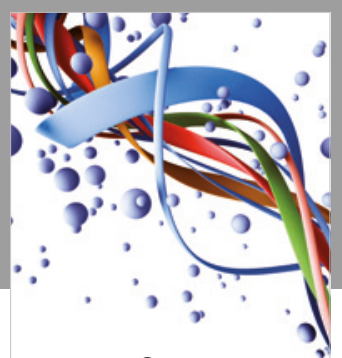

Scientifica

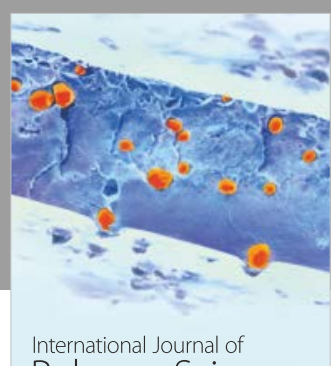

Polymer Science

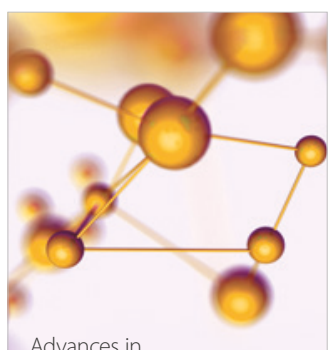

Physical Chemistry
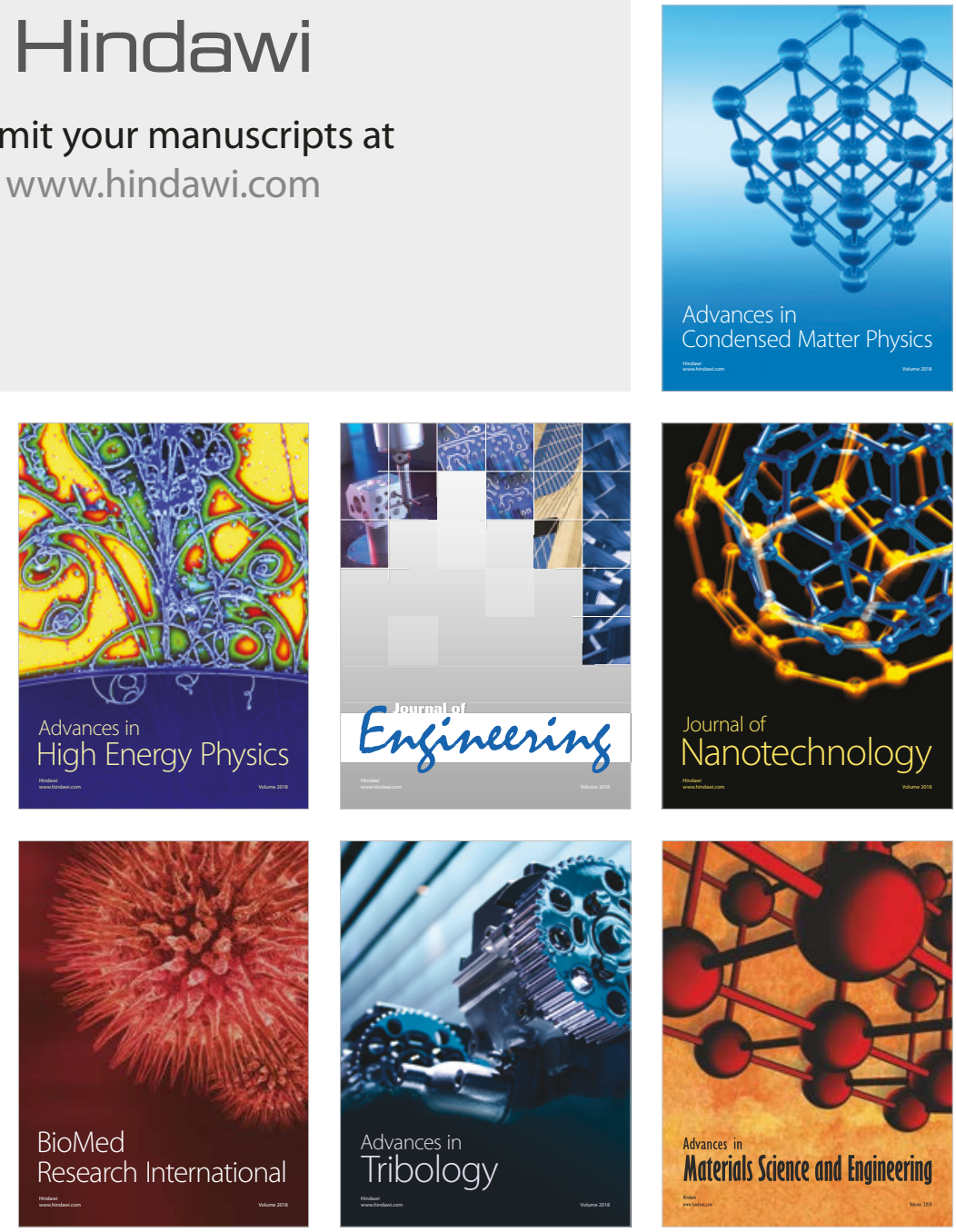